\title{
Simulation of Angle and Energy Resolved Fluxes of Escaping Neutral Particles from Fusion Plasmas with an Isotropic Ion Distribution
}

\author{
Evgeny A. VESHCHEV, Pavel R. GONCHAROV, Tetsuo OZAKI, Shigeru SUDO \\ and LHD Experimental Group \\ National Institute for Fusion Science, Toki, 509-5292, Japan
}

(Received 9 November 2007 / Accepted 14 March 2008)

\begin{abstract}
Multidirectional diagnostics employing high-resolution atomic energy spectrometers $[1,2]$ are being used to study the ion component heating mechanisms and fast ion confinement in helical plasmas. Since the natural atomic flux source is not localized in contrast to the pellet charge exchange [3,4] or diagnostic neutral beam methods [5], the correct interpretation of such measurements in a complex toroidally asymmetric geometry requires careful numerical modeling of the neutral flux formation and knowledge of the charge exchange target distributions, relevant cross-sections, and the magnetic surface structure. The measured neutral flux calculation scheme for LHD geometry was given in [6], and the influence of the geometry effect on the interpretation of measured data was shown. The current method was applied for the simulation of the experimental signal of the angularresolved multi-sightline neutral particle analyzer (ARMS-NPA) [1] along it's 20 sightlines in the LHD geometry configuration. In order to check the influence of the geometry effect on the interpretation of experimental results, calculations were conducted for the isotropic Maxwellian plasma-ion-energy probability density function. The behavior of the calculated and experimental ion spectra from neutral beam injector (NBI) is discussed.
\end{abstract}

(c) 2008 The Japan Society of Plasma Science and Nuclear Fusion Research

Keywords: neutral particle, fast ion, angle resolved measurement, fast particle flux simulation, ion distribution, angular distribution, energy resolved measurement, distribution function, diagnostic, analyzer

DOI: $10.1585 /$ pfr.3.S1035

\section{Introduction}

Beside high-resolution energy spectra of fast particles, a multi-directional neutral particle analyzer can also provide information about their angular distribution during scans of the plasma column in the tangential direction. A vertical scan of the plasma column by the multi-directional diagnostic can provide information about the radial distribution of the fast particles. Such measurements are required for understanding the fast ions behavior in plasmas, checking the fast particle loss-cone presence, studying the heating mechanisms, etc. Such information is important for the fast particle confinement and ignition of the future fusion reactor. For this purpose, the novel ARMS-NPA has been developed [1], which can measure plasma in the vertical and tangential directions. As the magnetic field geometry of an LHD has a very complex 3D structure, simulations of the experimental signal of the flux of the fast particles is required with a consideration of the attenuation of fast particles on the way to the detector due to the charge exchange, and the influence path length of the particle along every scanning chord to understand the influence of geometry of the measurements on the angular fast particle distribution. The difference in the geometry of the measurements can be clearly seen in Fig. 1, in which the top view of the detector sightlines versus the LHD plasma column is shown, and in Fig. 2, where the vertical crosssection plane of every detector sightline is shown. Detector 1 is in the tangential direction and detector 20 is almost in the perpendicular direction. The sheaf of sightlines was adjusted in such a way that all the channels to be observed were as close as possible to the central region of the plasma.

\section{Calculation Scheme}

The escaping neutral flux formulation has been made in [6] for passive diagnostics. The atomic flux $\Gamma(E, \vartheta)\left[\operatorname{erg}^{-1} \mathrm{~s}^{-1}\right]$ measured along the sightline with the normalized minor radius $\rho$ (where $E$ is the energy of the particles, and $\vartheta$ is the pitch-angle between the diagnostic sightline and the magnetic field) can be expressed as follows:

$$
\begin{aligned}
\Gamma(E, \vartheta)= & \exp \left\{\int_{\rho_{\min }}^{1} \frac{Q^{-}\left(\rho^{\prime}\right) \mathrm{d} \rho^{\prime}}{\lambda_{\mathrm{mfp}}\left(E, \rho^{\prime}\right)}\right\} \frac{\Omega S_{a}}{4 \pi} \int_{\rho_{\min }}^{1} g(E, \vartheta, \rho) \\
& \times\left[Q^{+}(\rho) \exp \left\{-\int_{\rho_{\min }}^{\rho} \frac{Q^{+}\left(\rho^{\prime}\right) \mathrm{d} \rho^{\prime}}{\lambda_{\mathrm{mfp}}\left(E, \rho^{\prime}\right)}\right\}\right.
\end{aligned}
$$




$$
-Q^{-}(\rho) \exp \left\{-\int_{\rho_{\min }}^{\rho} \frac{Q^{-}\left(\rho^{\prime}\right) \mathrm{d} \rho^{\prime}}{\lambda_{\mathrm{mfp}}\left(E, \rho^{\prime}\right)}\right\} \mathrm{d} \rho,
$$

where $\Omega$ is the observable solid angle and $S_{\mathrm{a}}$ is the diagnostic aperture area. The functions $Q^{+}(\rho)=\mathrm{d} X / \mathrm{d} \rho>0$ and $Q^{-}(\rho)=\mathrm{d} X / \mathrm{d} \rho<0$ on the two intervals between $\rho=1$ and $\rho=\rho_{\text {min }}$ are obtained from the structure of the isolines $\rho=$ const that are known from a numerical solution

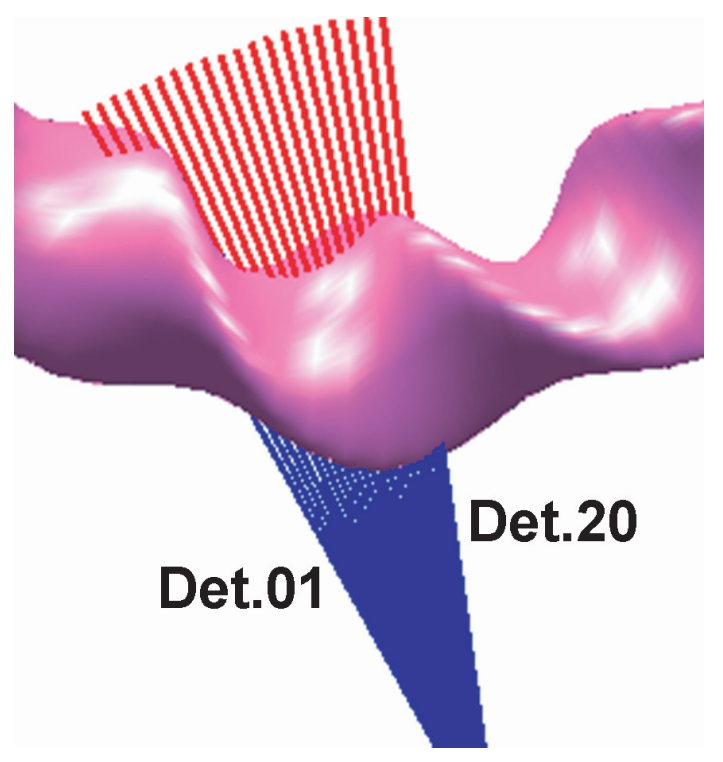

Fig. 1 Top view of the detector chords versus LHD plasma column. Blue color is the part of sightline close to the detector. of Grad Shafranov equation [7].

In order to check the geometry influence, the current formulation was applied for the simulation of the experimental signal along all 20 sightlines in the LHD geometry configuration. The simulation was performed for the isotropic Maxwellian plasma ion energy probability density function,

$$
f_{\mathrm{i}}^{(M)}(E, \rho)=\frac{2 \sqrt{E}}{\pi^{1 / 2} T_{\mathrm{i}}^{3 / 2}(\rho)} \exp \left(-E / T_{\mathrm{i}}(\rho)\right) .
$$

It has already been shown that the geometry effect may influence the fast particles spectra [6], but not as significantly as in the experiment. This could be due to the insignificant difference in the compared magnetic configurations with $R_{\mathrm{ax}}=3.6$ and $3.53 \mathrm{~m}$. Thus, the new calculations were conducted for magnetic axis configurations with a greater difference $\left(R_{\mathrm{ax}}=3.6\right.$ and $\left.3.9 \mathrm{~m}\right)$ for all 20 sightlines. The experimentally measurable $\Gamma(E, \vartheta)$ has been calculated for hydrogen plasmas based on the following radial profile assumptions:

$$
\begin{array}{ll}
n_{\mathrm{e}}(\rho)=n_{\mathrm{e}}(0)\left(1-\rho^{q}\right)^{s}, & n_{0}(\rho)=n_{0}(0) \exp \left(B \rho^{A}\right), \\
T_{\mathrm{i}}(\rho)=T_{\mathrm{i}}(0)\left(1-\rho^{x}\right)^{y}, & T_{\mathrm{e}}(\rho)=T_{\mathrm{e}}(0)\left(1-\rho^{u}\right)^{w},
\end{array}
$$

with the unknown values taken as free parameters. The values of the plasma components in the core are $n_{0}(0)=$ $10^{8} \mathrm{~cm}^{-3}, n_{\mathrm{e}}(0)=10^{13} \mathrm{~cm}^{-3}$, and $T_{\mathrm{e}}(0)=T_{\mathrm{i}}(0)=1 \mathrm{keV}$.

The results of the simulation can be seen in Fig. 3 . Both the cases demonstrate angular anisotropy due to the

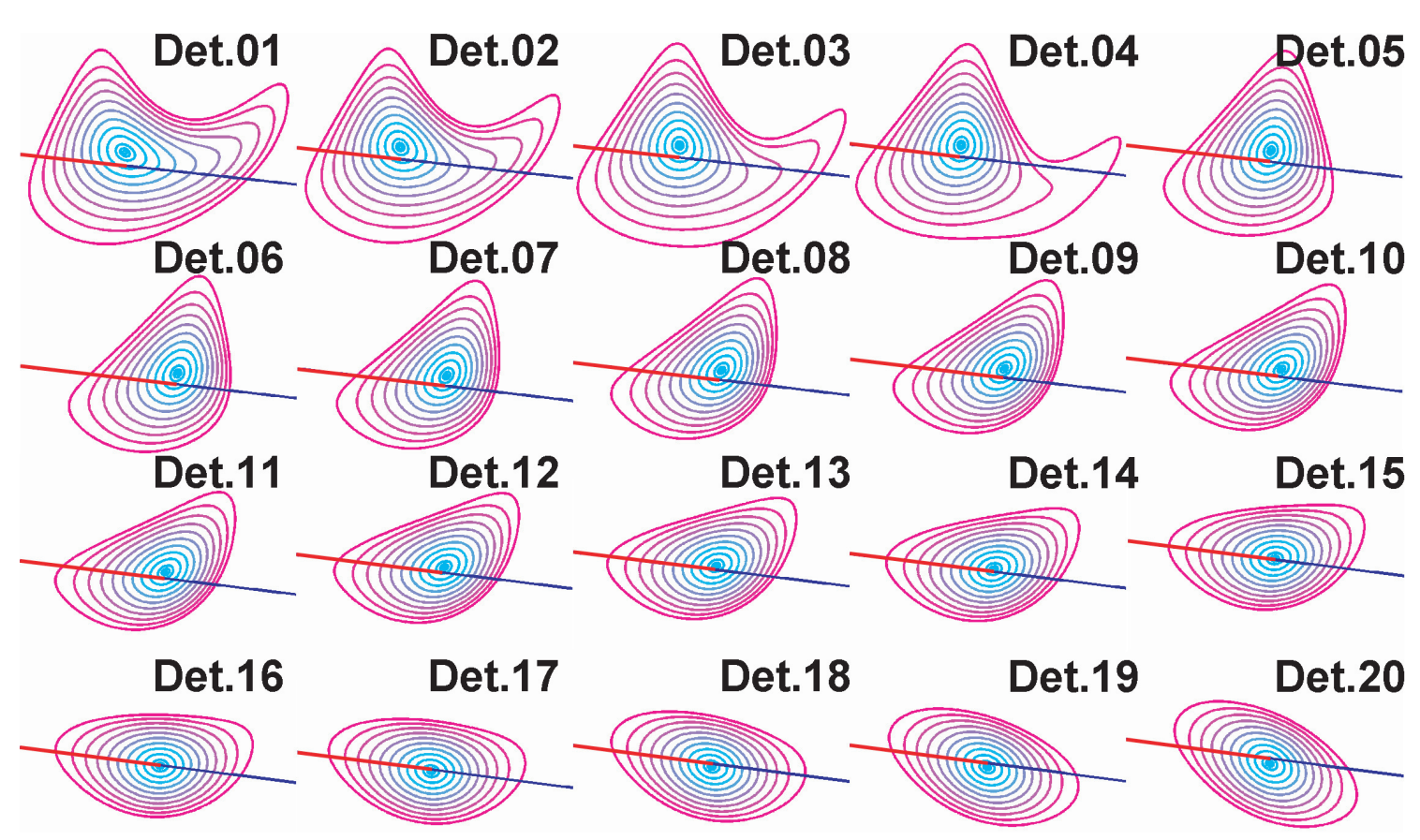

Fig. 2 Vertical cross-section plane of every ARMS-NPA sightline. Red color of the line correspond to the scanned part of plasma close to the LHD center. Blue color is the part of sightline close to the detector. Detector 1 corresponds to the most tangential direction and detector 20 is the direction close to the perpendicular one. 



Fig. 3 Calculated angular resolved spectra of fast particles a) for $R_{\mathrm{ax}}=3.6 \mathrm{~m}$ magnetic axis position and b) for $R_{\mathrm{ax}}=3.9 \mathrm{~m}$ magnetic axis position.
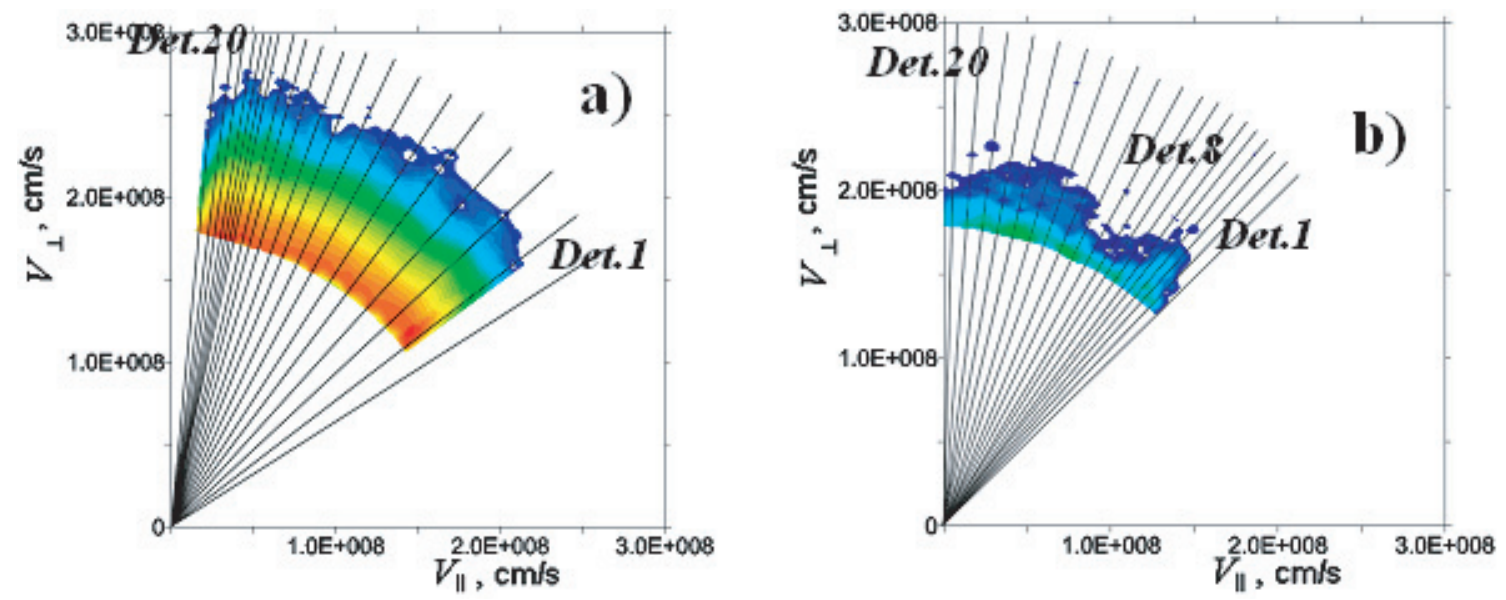

Fig. 4 Experimental results of angular distribution of fast particles a) for $R_{\mathrm{ax}}=3.6 \mathrm{~m}$ magnetic axis position and b) for $R_{\mathrm{ax}}=3.9 \mathrm{~m}$ magnetic axis position.

geometry influence, and in both the cases, fast particle population is reduced in the perpendicular region.

\section{Experimental Results}

Angular resolved measurements were conducted for both the magnetic axis configurations $\left(R_{\mathrm{ax}}=3.6\right.$ and $3.9 \mathrm{~m})$. Angular resolved spectra are plotted in Fig. 4. Both the cases demonstrate angular anisotropy and a reduction in the fast particle population in the perpendicular direction. In order to understand if such a behavior of fast particle spectra is due to the geometry effect, it must be extracted from the experimental data. The geometry of the measurements influences only the relative values of the fast particles, but not the shape of spectra; therefore, for the geometry effect correction, it is sufficient to divide the experimental spectra along every sightline by the relative values obtained from the calculation results, i.e., the corrected flux for every sightline is given by

$$
\Gamma_{\mathrm{N}}(E, \vartheta)_{\text {corrected }}=\frac{\Gamma_{\mathrm{N}}(E, \vartheta)_{\text {exp erimental }}}{\Gamma_{\mathrm{N}}\left(E_{\min }, \vartheta\right)_{\text {calculated }}},
$$

where $N$ is the number of the detector sightline, and $E_{\min }$ is the minimum energy of the fast particles measured by the ARMS-NPA $\left(E_{\min }=18 \mathrm{keV}\right)$.

The angular resolved spectra plotted in Fig. 5 represent experimental data of the angular distribution of the fast particles after the geometry effect correction for $R_{\mathrm{ax}}=3.6$ and $3.9 \mathrm{~m}$. Both cases still demonstrate angular anisotropy. Fast particle population in the $R_{\mathrm{ax}}=3.6 \mathrm{~m}$ configuration measured along four perpendicular sightlines are plotted in Fig. 6, which demonstrate a reduction of the spectra. The case of $R_{\mathrm{ax}}=3.9 \mathrm{~m}$ in addition to the reduction of the fast particle flux in the perpendicular direction (Fig. 5(b)) still demonstrates the drop in the fast particle population in the region of the 8th channel. Such a behavior can be due to the presence of the loss-cone region. 



Fig. 5 Experimental data of angular distribution of fast particles after the geometry effect correction a) for $R_{\mathrm{ax}}=3.6 \mathrm{~m}$ magnetic axis position and $\mathrm{b}$ ) for $R_{\mathrm{ax}}=3.9 \mathrm{~m}$ magnetic axis position.



Fig. 6 Fast particle spectra for four of the sightlines close to perpendicular direction (sightline 20 is the most perpendicular one) during perpendicularly-injecting NBI4 operation the case of $R_{\mathrm{ax}}=3.6 \mathrm{~m} B_{\mathrm{T}}=2.75 \mathrm{~T}$ magnetic field after the geometry effect correction.

\section{Conclusion}

Although the simulation results demonstrate the reduction of the fast particle population in the perpendicular direction, the geometry effect cannot completely explain the drop in the fast particle population observed along most perpendicular diagnostic sightlines. Thus, the possibility of a fast particle loss-cone region near the perpendicular region may exist in the LHD.

[1] E.A. Veshchev, T. Ozaki et al., Rev. Sci. Instrum. 77, 10F129-1 (2006).

[2] J.F. Lyon, P.R. Goncharov et.al., Rev. Sci. Instrum. 74, 1873 (2003).

[3] P.R. Goncharov, T. Ozaki et al., Rev. Sci. Instrum. 77, 10F119-1 (2006).

[4] P.R. Goncharov, T. Saida et.al., Rev. Sci. Instrum. 74, 1869 (2003).

[5] H. Matsushita and K. Ida et al., Rev. Sci. Instrum. 75, 3607 (2004).

[6] E.A. Veshchev, P.R. Goncharov et al., Rev. Sci. Instrum. 77, 10F116-1 (2006).

[7] L.L. Lao, S.P. Hirshman et al., Phys. Fluids 24, 1431 (1981). 